\section{Enseñar Bioética a los futuros licenciados en salud}

\section{Giornelli, María Gabriela}

Universidad Juan Agustín Maza - Mendoza - Argentina. Email:gabgiornelli@gmail.com

PALAVRAS-CHAVE: Enseñanza - Bioética - Licenciados en salud

Introducción: A partir del año 2015 la Facultad de Farmacia y Bioquímica de la Universidad Maza comenzó a dictar un ciclo de complementación curricular para que técnicos en diferentes áreas de la salud (Hemoterapia, Anatomía patológica, Instrumentación quirúrgica y Laboratorio clínico) alcancen el grado de licenciatura. En el plan de estudios aprobado por el Ministerio de Educación de la Nación se incluye la actividad curricular Ética y bioética. Se dictó en forma presencial pero en el espacio de la Bioética se fortaleció el proceso de enseñanza - aprendizaje a través del complemento virtual de la cátedra. En el año 2016 se sumaron alumnos de las carreras de Terapia Ocupacional y Radiología que dicta la Facultad de Kinesiología y Fisioterapia de la misma universidad. Desde el año 2017, el dictado es completamente a través de la plataforma virtual de la Universidad, sumándose alumnos de Anestesiología. Este curso tiene como objetivo principal dar a conocer y comprender el alcance e implicancias de los antecedentes, principios y temas fundamentales en materia de Bioética y su relación con el desempeño profesional en salud, en concordancia con normativas éticas provinciales, nacionales e internacionales. Algunos de sus objetivos particulares son: propiciar un ámbito de debate para generar capacidad crítica y analítica en los alumnos y fomentar la divergencia de opiniones y posturas basadas en el conocimiento, a partir del respeto y la empatía entre los alumnos y los profesores. En este trabajo se pretende relevar en los comentarios de los alumnos en los foros virtuales, la importancia que los mismos le dieran a la inclusión de contenidos bioéticos para un mejor desempeño en sus tareas asistenciales. Metodología: La metodología es de tipo exploratoria. Se relevaron las opiniones vertidas en un foro de discusión por los alumnos de las cuatro cohortes (2015 a 2018) del ciclo de complementación curricular para técnicos en diferentes áreas de la salud. Se planteó en el foro la pregunta: Desde su experiencia laboral o a partir de sus expectativas, ¿en qué aspectos o temas su trabajo se compromete o relaciona con la Bioética? Resultados: Analizados los comentarios vertidos por los alumnos en el foro de discusión, se encontró que, sobre un total de 433 alumnos se encontró que la mayoría encontró que la bioética atravesaba todas las actividades, tareas y/o funciones de su desempeño laboral. Discusión: De la lectura de 300 comentarios vertidos en el foro de discusión (de los cuales se seleccionan solo algunos a modo de ejemplo), se pone de manifiesto la necesidad de formar conciencias éticas y bioéticas para mejorar la relación personal (técnico) o profesional de la salud/ paciente, logrando un trato que anteponga valores y principios frente a las decisiones que deben tomarse. Conclusiones: El espacio curricular para el aprendizaje de la bioética en los ciclos de complementación curricular para licenciados en salud debería impregnar toda la formación técnica y de habilidades del alumno y no sólo limitarse a la enseñanza en el aula. La enseñanza de la bioética exige un compromiso de parte del docente.

AGRADECIMENTOS. A mis alumnos del ciclo de complementación curricular, por el interés, dedicación, tenacidad y perseverancia manifestados a lo largo del cursado para superarse en su formación personal, impactando en el ámbito laboral, profesional y social. Y a la Universidad Juan Agustín Maza por el apoyo y la confianza que me brindan para el desarrollo de mi tarea docente.

\section{REFERÊNCIAS}

[1] Correa, L., \& Javier, F. (2008). Enseñar bioética: cómo trasmitir conocimientos, actitudes y valores. Acta bioethica, 14(1), 11-18.

[2] Hodelín Tablada, R. (2007). La ética y la bioética en el pregrado ponencia introductoria: la enseñanza de la bioética en el pregrado. Reflexiones sobre un problema inconcluso. Revista Habanera de Ciencias Médicas, 6, 0-0.

[3] Blasco, P. G., De Otálora, M. S. D., Pastushenko, J., \& Trota, R. A. (2009). ¿Cómo enseñar bioética en el pregrado? Reflexiones sobre experiencias docentes. Atención primaria, 41(2), 103-108.

[4] Rennó Junqueira, Cilene, Tavares da Silva, Priscila Machado, Rennó Junqueira, Simone, \& Ramos, Dalton Luiz de Paula. (2012). O ensino de bioética: avaliação discente por meio de fóruns de discussão na Internet. Acta bioethica, 18(1), 93-100. $\quad$ https://dx.doi.org/10.4067/S1726569X2012000100008

[5] Moreto, Graziela, Bariani, Daniela B., Pinheiro, Thais Raquel, Altisent, Rogelio, \& González-Blasco, Pablo. (2008). Una nueva metodología docente en bioética: experiencias con la aplicación del portafolio a estudiantes de medicina en brasil. Persona y bioética, 12(2), 132-144. 